\title{
INTERPOLATION AND FUNCTIONS ANALYTIC INTERIOR TO THE UNIT CIRCLE*
}

\author{
BY \\ J. L. WALSH
}

Introduction. The present paper is a contribution to the study of the following problem. Given points $\beta_{1}, \beta_{2}, \ldots$ interior to the unit circle, and functional values $\gamma_{1}, \gamma_{2}, \cdots$. Does there exist a function $f(z)$ analytic for $|z|<1$ which takes on the values $\gamma_{n}$ in the respective points $\beta_{n}$ ? Is this function unique, if it exists? What representation can be given for the function? When can it be represented by Cauchy's integral taken over the unit circle?

This problem has recently been considered by various writers, notably Carathéodory and Fejér, Gronwall, I. Schur, Pick, Denjoy, and R. Nevanlinna, $\nmid$ who study primarily functions $f(z)$ which are uniformly bounded for $|z|<1$. The present paper studies particularly the case that $f(z)$ can be represented by the integral

$$
f(z)=\frac{1}{2 \pi i} \int_{C} \frac{f_{1}(t) d t}{t-z}, \quad C:|z|=1,
$$

where $f_{1}(t)$ is integrable together with its square or more generally merely integrable on $C$. The case that $f_{1}(t)$ is integrable together with its square on $C$ has previously been studied by Malmquist, $\ddagger$ but only under the assumptions that the product $\prod\left|\beta_{n}\right|$ diverges, that every $\beta_{n}$ is different from zero, and that the $\beta_{n}$ are all distinct. The present methods of study of the case that $\prod\left|\beta_{n}\right|$ diverges are related to those of Malmquist; most of the results of II below are due to him or are generalizations of his results. Our results of III and IV are in the main new, however, so far as the writer is aware.

The present paper gives what can be considered a complete solution of this problem of interpolation so far as concerns functions $f(z)$ which can be represented by (1) where $f_{1}(t)$ is integrable together with its square on $C$. Rapidly sketched, our main result is the following. An arbitrary function $f(z)$ analytic for $|z|<1$ has a formal expansion

* Presented to the Society, March 25, 1932; received by the editors March 16, 1932.

$\dagger$ Detailed references are given by R. Nevanlinna, Annales Academiae Scientiarum Fennicae, vol. 32 (1929).

$\ddagger$ Comptes Rendus du Sixième Congrès (1925) des Mathématiciens Scandinaves (Kopenhagen, 1926), pp. 253-259. I am indebted for this reference to Professor Einar Hille.

Malmquist does not actually make the restriction $\beta_{n} \neq 0$, but that is tacitly implied. 
(2) $f(z)=\frac{a_{0}}{1-\bar{\beta}_{1} z}+\frac{a_{1}\left(z-\beta_{1}\right)}{\left(1-\bar{\beta}_{1} z\right)\left(1-\bar{\beta}_{2} z\right)}+\frac{a_{2}\left(z-\beta_{1}\right)\left(z-\beta_{2}\right)}{\left(1-\bar{\beta}_{1} z\right)\left(1-\bar{\beta}_{2} z\right)\left(1-\bar{\beta}_{3} z\right)}+\cdots$

in which the coefficients $a_{n}$ are found (in terms of the $\beta_{k}$ and the values $f\left(\beta_{k}\right)$ ) by interpolation in the points $\beta_{n}$. This formal expansion exists (independently of any function $f(z)$ ) and can be determined from given functional values $\gamma_{n}$ and the points $\beta_{n}$ alone. $A$ necessary and sufficient condition that a function $f(z)$ exist which takes on the given values $\gamma_{n}$ in the given points $\beta_{n}$ and which can be represented for $|z|<1$ by (1) where $f_{1}(t)$ is integrable together with its square on $C$, is that the formal expansion (2) have coefficients $a_{n}$ such that the series

$$
\sum \frac{\left|a_{n-1}\right|^{2}}{1-\left|\beta_{n}\right|}
$$

converges. If this condition is fulfilled, equation (2) represents for $|z|<1$ a function $f(z)$ of the kind required.

\section{Preliminary Results}

1. Series of interpolation. We shall be particularly concerned with series of special form of which the following is typical:

$$
f(z)=a_{0}+a_{1} \frac{z-\beta_{1}}{z-\alpha_{1}}+a_{2} \frac{\left(z-\beta_{1}\right)\left(z-\beta_{2}\right)}{\left(z-\alpha_{1}\right)\left(z-\alpha_{2}\right)}+\cdots,
$$

where the $\alpha_{n}$ are distinct from the $\beta_{i}$. If the function $f(z)$ is defined in the distinct points $\beta_{n}$, a formal expansion (1.1) of $f(z)$ exists, for the coefficients $a_{n}$ can be uniquely determined in terms of the values $f\left(\beta_{i}\right)$. If in (1.1) we set $z=\beta_{1}$, we have

$$
f\left(\beta_{1}\right)=a_{0} .
$$

If in (1.1) we now set $z=\beta_{2}$, we have

$$
f\left(\beta_{2}\right)=a_{0}+a_{1} \frac{\beta_{2}-\beta_{1}}{\beta_{2}-\alpha_{1}},
$$

so that $a_{1}$ is determined. By continuation in this way, we see that the coefficients $a_{n}$ are successively uniquely determined.

If the $\beta_{n}$ are not all distinct, we assume the existence of suitable derivatives of $f(z)$ in the multiple points $\beta_{n}$. We make the convention that at a point $z=\beta$ at which precisely $k$ of the points $\beta_{n}$ coincide, equation (1.1) shall be interpreted as implying the validity of $(1.1)$ and its first $(k-1)$ derived equations for the value $z=\beta$. With this convention, the coefficients $a_{n}$ are all uniquely determined whether or not the $\beta_{n}$ are all distinct. If $a_{0}, a_{1}, \cdots$, 
$a_{n-1}$ are known, and if precisely $m$ of the points $\beta_{1}, \beta_{2}, \cdots, \beta_{n}$ are equal to $\beta_{n+1}$, the $m$ th derived equation of (1.1) for $z=\beta_{n+1}$ determines $a_{n}$.

The values $\alpha_{n}=\infty, \beta_{n}=\infty$ are not included in (1.1) according to a literal interpretation of that equation, but we expressly admit them nevertheless as possible values, although in the present paper $\beta_{n}=\infty$ is not to be used.

The formal expansion (1.1) is surely valid in the points $\beta_{n}$, and under suitable circumstances it can be shown* that if $f(z)$ is analytic in a certain region and the $\alpha_{n}$ and $\beta_{n}$ are suitably located, then the formal expansion (1.1) of $f(z)$ converges to $f(z)$ in some region. We shall later be concerned with some special results of this sort.

2. Functions analytic interior to the unit circle. If the function $f(z)$, analytic interior to $C:|z|=1$, can be represented by the integral

$$
f(z)=\frac{1}{2 \pi i} \int_{C} \frac{f_{1}(t) d t}{t-z}, \quad|z|<1,
$$

where $f_{1}(t)$ together with its $p$ th power $(p \geqq 1)$ is integrable on $C$, then $f(z)$ will be said to belong to class $E_{p}$. We shall be particularly concerned in the present paper with the classes $E_{1}$ (denoted henceforth by $E$ ) and $E_{2}$. If $f(z)$ can be represented by (2.1) for $|z|<1$, where $f_{1}(t)$ is limited on $C$, then $f(z)$ will be said to belong to class $E^{\prime}$.

We use the notation (2.1) throughout the present paper; that is, whenever $f(z)$ is given of class $E_{p}$, we write (2.1), where $f_{1}(t)$ together with its $p$ th power is integrable on $C$.

The function $f_{1}(z)$ which appears in $(2.1)$ is naturally not uniquely determined on $C$ by the knowledge of $f(z)$ interior to $C$. Indeed, there is a large class of functions $\phi_{1}(z)$, including any polynomial in $1 / z$ without constant term, such that we have

$$
\frac{1}{2 \pi i} \int_{C} \frac{\phi_{1}(t) d t}{t-z}=0, \quad|z|<1 .
$$

If (2.1) is valid, we have obviously

$$
f(z)=\frac{1}{2 \pi i} \int_{C} \frac{f_{1}(t)+\phi_{1}(t)}{t-z} d t, \quad|z|<1 .
$$

It may occur for a function $f(z)$ given analytic for $|z|<1$, that its properties for $|z|<1$ enable the determination of a function $f_{1}(z)$ defined in some way on $C$ as the boundary values of $f(z)$ as $z$ (interior to $C$ ) approaches $C$

* Angelescu, Bulletin, Académie Roumaine, vol. 9 (1925), pp. 164-168.

Walsh, these Transactions, vol. 34 (1932), pp. 22-74; Proceedings of the National Academy of Sciences, vol. 18 (1932), pp. 165-171. 
along a radius. Thus if $f(z)$ is bounded interior to $C$, these boundary values $f_{1}(z)$ exist almost everywhere on $C$ (Fatou) and (2.1) is valid. The function $f_{1}(z)$ is limited on $C$, so $f(z)$ (assumed merely bounded interior to $C$ ) is of class $E_{p}$ for every $p \geqq 1$

Moreover, if the function

$$
f(z)=a_{0}+a_{1} z+a_{2} z^{2}+\cdots,|z|<1,
$$

is such that $\sum\left|a_{n}\right|^{2}$ converges, the boundary values $f_{1}(z)$ of $f(z)$ also exist almost everywhere on $C$ for radial approach, the function $f_{1}(z)$ is integrable together with its square on $C$, and (2.1) is valid.*

Reciprocally, let (2.1) be valid, where $f_{1}(z)$ is integrable together with its square on $C$; we have

$$
f(z)=\frac{1}{2 \pi i} \int_{C} \frac{f_{1}(t) d t}{t-z}=\frac{1}{2 \pi i} \int_{C} f_{1}(t) d t\left[\frac{1}{t}+\frac{z}{t^{2}}+\frac{z^{2}}{t^{3}}+\cdots\right],|z|<1
$$

it is allowable to integrate this series term by term. That is, (2.2) is valid, where we have

$$
a_{n}=\frac{1}{2 \pi i} \int_{C} \frac{f_{1}(t) d t}{t^{n+1}}=\frac{1}{2 \pi} \int_{C} f_{1}(t) \bar{t}^{n}|d t|,
$$

so (2.2) is the formal expansion of $f_{1}(z)$ on $C$ in terms of the orthogonal set 1 , $z, z^{2}, \cdots$. This set can readily be normalized on $C$, and it follows that the series $\sum\left|a_{n}\right|^{2}$ converges. Then the boundary values of $f(z)$ exist almost everywhere on $C$ for radial approach, and other properties follow from the theorem of F. and M. Riesz just mentioned. But in (2.3) the function $f_{1}(z)$ naturally need not represent the boundary values of $f(z)$ on $C$.

A necessary and sufficient condition that we have

$$
\frac{1}{2 \pi i} \int_{C} \frac{f_{1}(t) d t}{t-z}=\frac{1}{2 \pi i} \int_{C} \frac{f_{2}(t) d t}{t-z},|z|<1,
$$

where $f_{1}(t)$ and $f_{2}(t)$ are integrable on $C$, is that we have

$$
\int_{C} \frac{f_{1}(t) d t}{t^{n}}=\int_{C} \frac{f_{2}(t) d t}{t^{n}} \quad(n=1,2,3, \cdots)
$$

for we can write

$$
\int_{C} \frac{f_{1}(t)-f_{2}(t)}{t-z} d t=\int_{C}\left[f_{1}(t)-f_{2}(t)\right]\left[\frac{1}{t}+\frac{z}{t^{2}}+\frac{z^{2}}{t^{3}}+\cdots\right] d t, \quad|z|<1 .
$$

\footnotetext{
* F. and M. Riesz, Compte Rendu du Quatrième Congrès (1916) des Mathématiciens Scandi-
} naves (Uppsala, 1920), pp. 27-44. 
A necessary and sufficient condition for the identical vanishing of the righthand member, $|z|<1$, since the series can be integrated term by term is precisely (2.5).

3. Blaschke products. Another preliminary result is the well known convergence of the Blaschke product, namely that if we have $\left|\beta_{n}\right|<1$, and if the product $\Pi\left|\beta_{n}\right|$ converges, then the product

$$
\Pi \frac{z-\beta_{n}}{z-\frac{1}{\bar{\beta}_{n}}}=\prod \frac{z-\beta_{n}}{\bar{\beta}_{n} z-1} \bar{\beta}_{n}
$$

converges for $|z|<1$, uniformly for $|z| \leqq r<1$, and represents a function whose modulus for $|z|<1$ is less than $\prod\left|\beta_{n}\right|$.

Indeed (compare (5.2) below), a necessary and sufficient condition for the convergence of (3.1) for even a particular value of $z$ interior to $C$ is the convergence of $\Pi\left|\beta_{n}\right|$.

Both forms in (3.1) fail unless all the $\beta_{n}$ are different from zero. If a particular $\beta_{n}$ vanishes, the corresponding factor in both forms of (3.1) is to be replaced by $z$ itself.

A necessary and sufficient condition for the convergence of $\Pi\left|\beta_{n}\right|$ is the convergence of the series

$$
\sum\left(1-\left|\beta_{n}\right|\right)
$$

as is well known.

In the proof of the following theorem and indeed in much of our later work, we find it convenient to assume $\beta_{n} \neq 0$; the reader can easily make the requisite modifications to remove this restriction.

THEOREM I. If the product $\Pi\left|\beta_{n}\right|$, or what amounts to the same, if the product

$$
B(z)=\Pi\left(\frac{z-\beta_{n}}{\bar{\beta}_{n} z-1} \bar{\beta}_{n}\right),\left|\beta_{n}\right|<1,|z|<1,
$$

converges, and if the Fatou boundary values on $C$ (defined almost everywhere on $C)$ are denoted by $B(z)$, then the sequence

$$
B_{n}(z)=\prod\left(\frac{z-\beta_{n}}{\bar{\beta}_{n} z-1} \bar{\beta}_{n}\right)
$$

converges in the mean (of order two) to $B(z)$ on $C$.

We prove first that the sequence $B_{n}(z)$ converges in the mean on $C$ and shall later identify the limit function (defined almost everywhere on $C$ ) with the Fatou boundary values of $B(z)$. 
A necessary and sufficient condition for the convergence in the mean of $B_{n}(z)$ on $C$ is the approach to zero with $1 / n$ of

$$
\int_{C}\left|B_{n+k}(z)-B_{n}(z)\right|^{2}|d z|
$$

and this expression, by virtue of the equation

$$
\left|\frac{z-\beta_{n}}{\bar{\beta}_{n} z-1}\right|=1, z \text { on } C
$$

can be written

$$
\begin{aligned}
& \left|\beta_{1} \beta_{2} \cdots \beta_{n}\right|^{2} \\
& \times \int_{C}\left|\frac{\left(z-\beta_{n+1}\right)\left(z-\beta_{n+2}\right) \cdots\left(z-\beta_{n+k}\right)}{\left(\bar{\beta}_{n+1} z-1\right)\left(\bar{\beta}_{n+2} z-1\right) \cdots\left(\bar{\beta}_{n+k} z-1\right)} \bar{\beta}_{n+1} \bar{\beta}_{n+2} \cdots \bar{\beta}_{n+k}-1\right|^{2} \mid
\end{aligned}
$$

We multiply the expression between vertical bars in the integrand by its conjugate and integrate, setting $|d z|=d z /(i z)$. We notice immediately the relation

$$
\begin{aligned}
& \int_{C} \frac{\left(z-\beta_{n+1}\right)\left(z-\beta_{n+2}\right) \cdots\left(z-\beta_{n+k}\right)}{\left(\bar{\beta}_{n+1} z-1\right)\left(\bar{\beta}_{n+2} z-1\right) \cdots\left(\bar{\beta}_{n+k} z-1\right)} \bar{\beta}_{n+1} \bar{\beta}_{n+2} \cdots \bar{\beta}_{n+k} \frac{d z}{i z} \\
& =2 \pi\left[\frac{\left(z-\beta_{n+1}\right)\left(z-\beta_{n+2}\right) \cdots\left(z-\beta_{n+k}\right)}{\left(\bar{\beta}_{n+1} z-1\right)\left(\bar{\beta}_{n+2} z-1\right) \cdots\left(\bar{\beta}_{n+k} z-1\right)} \bar{\beta}_{n+1} \bar{\beta}_{n+2} \cdots \bar{\beta}_{n+k}\right]_{z=0} \\
& =2 \pi\left|\beta_{n+1} \beta_{n+2} \cdots \beta_{n+k}\right|^{2} .
\end{aligned}
$$

That is, (3.4) reduces to

$$
2 \pi\left|\beta_{1} \beta_{2} \cdots \beta_{n}\right|^{2}\left[1-\left|\beta_{n+1} \beta_{n+2} \cdots \beta_{n+k}\right|^{2}\right] .
$$

The product $\left|\beta_{n+1} \beta_{n+2} \cdots \beta_{n+k}\right|$ approaches unity, by the convergence of $\Pi\left|\beta_{n}\right|$, so (3.5) and hence (3.4) approaches zero with $1 / n$. It remains to show that the function $B^{\prime}(z)$ to which the sequence $B_{n}(z)$ converges in the mean on $C$, is identical (almost everywhere) with the Fatou boundary values $B(z)$.

It follows from the Lemma about to be stated that we have

$$
\lim _{n \rightarrow \infty} \int_{C} B_{n}(z) z^{k} d z=\int_{C} B^{\prime}(z) z^{k} d z \quad(k=\cdots,-1,0,1,2, \cdots) .
$$

But if $C_{r}$ denotes the circle $|z|=r<1$, we have for these same values of $k$

$$
\lim _{n \rightarrow \infty} \int_{C} B_{n}(z) z^{k} d z=\lim _{n \rightarrow \infty} \int_{C_{r}} B_{n}(z) z^{k} d z=\int_{C_{r}} B(z) z^{k} d z=\int_{C} B(z) z^{k} d z .
$$


The equations

$$
\int_{C} B^{\prime}(z) z^{k} d z=\int_{C} B(z) z^{k} d z \quad(k=\cdots,-1,0,1,2, \cdots)
$$

are valid for the function $B^{\prime}(z)$, which by its genesis is known to be integrable together with its square on $C$, and for the bounded function $B(z)$. It follows that the two functions have the same Fourier coefficients (for $0 \leqq \theta \leqq 2 \pi$, $\left.z=e^{i \theta}\right)$, and hence that we have

$$
B(z)=B^{\prime}(z)
$$

almost everywhere on $C$.

The following lemma has just been applied, and will be needed as well in our later work. The easy proof of the lemma may be left to the reader. A proof is conveniently based on the fact that from any infinite sequence of the $\phi_{n}(z)$ can be extracted a subsequence which converges essentially uniformly on $C$ to the function $\phi(z)$.

Lемма. If the functions $\phi_{n}(z)$ are uniformly limited and converge in the mean on $C$ to the function $\phi(z)$, then for an arbitrary function $F(z)$ integrable on $C$ we have

$$
\lim _{n \rightarrow \infty} \int_{C} F(z) \phi_{n}(z)|d z|=\int_{C} F(z) \phi(z)|d z|
$$

It will be noticed too that if $F(z)$ contains a parameter $\lambda$ and if the inequalities for $F(z)$ can be chosen uniformly with respect to $\lambda$, then (3.6) is valid uniformly with respect to $\lambda$.

4. Formal expansions. A development not precisely of form (1.1) but nevertheless having many properties in common with such developments is

$$
\begin{aligned}
f(z)=\frac{a_{0}}{1-\bar{\beta}_{1} z} & +a_{1} \frac{z-\beta_{1}}{\left(1-\bar{\beta}_{1} z\right)\left(1-\bar{\beta}_{2} z\right)} \\
& +a_{2} \frac{\left(z-\beta_{1}\right)\left(z-\beta_{2}\right)}{\left(1-\bar{\beta}_{1} z\right)\left(1-\bar{\beta}_{2} z\right)\left(1-\bar{\beta}_{3} z\right)}+\cdots,
\end{aligned}
$$

where we suppose $\left|\beta_{n}\right|<1$. For the present, equation (4.1) is to be considered only formally. If the function $f(z)$ is defined in the distinct points $\beta_{n}$, the coefficients $a_{n}$ can be uniquely determined, as with (1.1), so a formal expansion of $f(z)$ exists, surely valid in the points $\beta_{n}$. If the $\beta_{n}$ are not all distinct, we assume the existence of suitable derivatives of $f(z)$ in the multiple points $\beta_{n}$. We make the previous convention that at a point $z=\beta$ at which precisely $k$ of the points $\beta_{n}$ coincide, equation (4.1) shall be interpreted as implying the validity of (4.1) and its first $(k-1)$ derived equations for the value $z=\beta$. With 
this convention, the coefficients $a_{n}$ are all uniquely determined; compare our discussion of (1.1).

The case that $\beta_{n}$ is not assumed different from zero is in our discussion constantly different from the contrary case, as we have already noted. Equation (1.1) does not exclude this case. In particular, if we have $\beta_{1}=0$, equation (4.1) takes the form

$$
f(z)=a_{0}+a_{1} \frac{z}{1-\bar{\beta}_{2} z}+a_{2} \frac{z\left(z-\beta_{2}\right)}{\left(1-\bar{\beta}_{2} z\right)\left(1-\bar{\beta}_{3} z\right)}+\cdots,
$$

which is of form (1.1).

The functions

(4.3) $\frac{1}{1-\bar{\beta}_{1} z}, \frac{z-\beta_{1}}{\left(1-\bar{\beta}_{1} z\right)\left(1-\bar{\beta}_{2} z\right)}, \frac{\left(z-\beta_{1}\right)\left(z-\beta_{2}\right)}{\left(1-\bar{\beta}_{1} z\right)\left(1-\bar{\beta}_{2} z\right)\left(1-\bar{\beta}_{3} z\right)}, \ldots$,

to be denoted respectively by $\phi_{0}(z), \phi_{1}(z), \cdots$, are mutually orthogonal on C:

$$
\int_{C} \phi_{n}(z) \overline{\phi_{m}(z)}|d z|=0, \quad m \neq n ;
$$

we leave the verification to the reader. This orthogonality relation is valid without reference to the restrictions $\beta_{n} \neq 0$ or $\beta_{i} \neq \beta_{j}$.

A given function of class $E$

$$
f(z)=\frac{1}{2 \pi i} \int_{C} \frac{f_{1}(t) d t}{t-z}, \quad|z|<1,
$$

where $f_{1}(z)$ is integrable on $C$, has two distinct formal expansions of form (4.1), one a formal expansion of $f_{1}(z)$ on $C$ in terms of the orthogonal functions $\phi_{n}(z)$ and found by integration on $C$ :

$$
\int_{C} f_{1}(z) \overline{\phi_{n}(z)}|d z|=a_{n} \int_{C} \phi_{n}(z) \overline{\phi_{n}(z)}|d z|,
$$

and the other found by interpolation to $f(z)$ in the points $\beta_{n}\left[\left|\beta_{n}\right|<1\right]$.

ThEOREM II. If we have (4.4) satisfied, where $f_{1}(t)$ is integrable on $C$, then the formal expansion of $f_{1}(z)$ on $C$ in terms of the set (4.3) is identical with the formal expansion (4.1) found by interpolation to the function $f(z)$ in the points $\beta_{n}$.

We prove Theorem II first in the case that $f(z)$ is analytic for $|z| \leqq 1$. Let the $a_{k}$ in (4.1) be determined by interpolation. Then the function 


$$
\begin{aligned}
F(z)=f(z) & -\frac{a_{0}}{1-\bar{\beta}_{1} z}-\frac{a_{1}\left(z-\beta_{1}\right)}{\left(1-\bar{\beta}_{1} z\right)\left(1-\bar{\beta}_{2} z\right)} \\
& -\cdots-\frac{a_{n}\left(z-\beta_{1}\right)\left(z-\beta_{2}\right) \cdots\left(z-\beta_{n}\right)}{\left(1-\bar{\beta}_{1} z\right)\left(1-\bar{\beta}_{2} z\right) \cdots\left(1-\bar{\beta}_{n+1} z\right)}
\end{aligned}
$$

vanishes in the points $\beta_{1}, \beta_{2}, \cdots, \beta_{n+1}$, by the method of determination of the $a_{k}$. It follows that $F(z)$ is orthogonal on $C$ to each of the functions $\phi_{0}(z)$, $\phi_{1}(z), \cdots, \phi_{n}(z)$; we find

$$
\int_{C} F(z) \overline{\phi_{m}} \overline{(z)}|d z|=\int_{C} F(z) \frac{\left(1-\bar{\beta}_{1} z\right)\left(1-\bar{\beta}_{2} z\right) \cdots\left(1-\bar{\beta}_{m} z\right) d z}{i\left(z-\beta_{1}\right)\left(z-\beta_{2}\right) \cdots\left(z-\beta_{m+1}\right)}, \quad(m=0,1, \cdots, n),
$$

and this integral vanishes by Cauchy's integral theorem. If we now multiply (4.6) through by $\overline{\phi_{n}(z)}|d z|$ and integrate over $C$, equation (4.5) (where $f(t)$ replaces $\left.f_{1}(t)\right)$ results. This proof is valid even if some or all of the points $\beta_{n}$ are multiple, thanks to the convention already made. It remains to point out that Theorem II is valid even if $f(\dot{z})$ is not analytic for $|z| \leqq 1$ but is given by (4.4), where $f_{1}(z)$ is integrable on $C$.

The function $\overline{\phi_{n}(z)}$ can be broken up into partial fractions whose denominators are the quantities $z-\beta_{k}(k=1,2, \cdots, n+1)$ (in case these $\beta_{k}$ are not all distinct, the denominators of the partial fractions contain powers of some $z-\beta_{k}$ higher than the first). Then $a_{n}$ as defined by (4.5) is a linear combination with coefficients involving the $\beta_{k}$ and $\bar{\beta}_{k}$ of the values $f\left(\beta_{1}\right), f\left(\beta_{2}\right)$, $\cdots, f\left(\beta_{m+1}\right)$ (if these $\beta_{k}$ are not all distinct, some of the values $f\left(\beta_{k}\right)$ are replaced by suitable derivatives of $f(z)$ for values $\left.z=\beta_{k}\right)$. We have already computed this linear combination for an arbitrary function $f(z)$ analytic on and within $C$, and shown that $a_{n}$ defined by (4.5) is the same as $a_{n}$ determined by interpolation, but in that computation the quantities $f\left(\beta_{k}\right)$ (and possible derivatives for $z=\beta_{k}$ of $f(z)$ ) enter into (4.5) only through Cauchy's integral (and the corresponding formulas found from it by differentiation). It follows that the validity of Cauchy's integral (4.4) (including the formulas found by differentiation of it) is sufficient to ensure the equality of $a_{n}$ found from (4.5) and $a_{n}$ found by interpolation, so the proof of Theorem II is complete.

If the function $f(z)$ of Theorem II is of class $E_{2}$, the sum of the first $n$ terms of this formal expansion (4.1) is simultaneously the unique function

$$
\frac{a_{0 n} z^{n-1}+a_{1 n} z^{n-2}+\cdots+a_{n-1, n}}{\left(1-\bar{\beta}_{1} z\right)\left(1-\bar{\beta}_{2} z\right) \cdots\left(1-\bar{\beta}_{n} z\right)}
$$

of best approximation to $f_{1}(z)$ on $C$ in the sense of least squares, and the unique function of form (4.7) which coincides with $f(z)$ in the points $\beta_{1}, \beta_{2}$, 
$\cdots, \beta_{n}$. Theorem II is more general than this remark, for if $f(z)$ is of class $E_{p}, p>2$, it may not be possible to refer to the best approximation to $f_{1}(z)$ on $C$ in the sense of least squares. Proof of the remark follows directly from Theorem II* and from the fact that each function (4.7) is the sum of $n$ terms of a series (4.1), and each sum of $n$ terms of (4.1) can be written in the form (4.7).

\section{THE PRODUCT $\Pi\left|\beta_{n}\right|$ DIVERGENT}

\section{Convergence of formal expansion. We now prove}

THEOREM III. If $f(z)$ is of class $E$ and hence can be represented by (4.4), where $f_{1}(t)$ is integrable on $C$, and if the product $\Pi\left|\beta_{n}\right|$ diverges, then the formal development (4.1) found either by expanding $f_{1}(z)$ on $C$ or by interpolation in the points $\beta_{n}$, converges to the function $f(z)$ for $|z|<1$, uniformly for $|z| \leqq r<1$.

Let $r_{n}(z)$ denote the sum of the first $n$ terms of the right-hand member of (4.1). Then we have

$$
\begin{aligned}
& f(z)-r_{n}(z) \\
& =\frac{1}{2 \pi i} \int_{C} f_{1}(t) \frac{\left(z-\beta_{1}\right)\left(z-\beta_{2}\right) \cdots\left(z-\beta_{n}\right)\left(1-\bar{\beta}_{1} t\right)\left(1-\bar{\beta}_{2} t\right) \cdots\left(1-\bar{\beta}_{n} t\right)}{\left(1-\bar{\beta}_{1} z\right)\left(1-\bar{\beta}_{2} z\right) \cdots\left(1-\bar{\beta}_{n} z\right)(t-z)\left(t-\beta_{1}\right)\left(t-\beta_{2}\right) \cdots\left(t-\beta_{n}\right)} d t .
\end{aligned}
$$

Formula (5.1) can be verified directly; if we apply Cauchy's integral (4.4), we see that $r_{n}(z)$ as defined by (5.1) is a rational function of $z$ whose denominator is of the form of the denominator of (4.6) and whose numerator is of the proper form; it is seen directly from (5.1) that $r_{n}(z)$ agrees with $f(z)$ in the points $z=\beta_{1}, \beta_{2}, \cdots, \beta_{n}$. These conditions determine $r_{n}(z)$ uniquely.

On $C$ the quantities

$$
\frac{1-\bar{\beta}_{n} t}{t-\beta_{n}}
$$

which appear in (5.1) all have the modulus unity. Thus we have for $|z| \leqq r<1$

$$
\left|f(z)-r_{n}(z)\right| \leqq\left|\frac{\left(z-\beta_{1}\right)\left(z-\beta_{2}\right) \cdots\left(z-\beta_{n}\right)}{\left(1-\bar{\beta}_{1} z\right)\left(1-\bar{\beta}_{2} z\right) \cdots\left(1-\bar{\beta}_{n} z\right)}\right| \frac{1}{2 \pi(1-r)} \int_{C}\left|f_{1}(t)\right||d t| .
$$

But for $|z| \leqq r<1,|\beta|<1$, we have

$$
\left|\frac{z-\beta}{1-\bar{\beta} z}\right| \leqq \frac{r+|\beta|}{1+|\beta| r} .
$$

* It is well known that the sum of the first $n$ terms of the formal expansion $f(z) \sim a_{1} \phi_{1}(z)+a_{2} \phi_{2}(z)+\cdots$

of a function integrable on $C$ together with its square in terms of the orthogonal set $\phi_{i}(z)$ is also the linear combination of the first $n$ functions $\phi_{i}(z)$ of best approximation to $f(z)$ on $C$ in the sense of least squares. See, for instance, Kowalewski, Determinantentheorie, Leipzig, 1909, p. 335. 
There follows the inequality for $|z| \leqq r<1$

$$
\begin{aligned}
& \left|\frac{\left(z-\beta_{1}\right)\left(z-\beta_{2}\right) \cdots\left(z-\beta_{n}\right)}{\left(1-\bar{\beta}_{1} z\right)\left(1-\bar{\beta}_{2} z\right) \cdots\left(1-\bar{\beta}_{n} z\right)}\right| \\
& \quad \leqq \frac{\left(r+\left|\beta_{1}\right|\right)\left(r+\left|\beta_{2}\right|\right) \cdots\left(r+\left|\beta_{n}\right|\right)}{\left(1+\left|\beta_{1}\right| r\right)\left(1+\left|\beta_{2}\right| r\right) \cdots\left(1+\left|\beta_{n}\right| r\right)}
\end{aligned}
$$

This right-hand member is less than unity and hence approaches zero with $1 / n$, for the divergence of $\prod\left|\beta_{n}\right|$ implies the divergence of $\sum\left(1-\left|\beta_{n}\right|\right)$ and hence the divergence of

$$
\sum\left(1-\frac{r+\left|\beta_{n}\right|}{1+\left|\beta_{n}\right| r}\right)=\sum \frac{\left(1-\left|\beta_{n}\right|\right)(1-r)}{1+\left|\beta_{n}\right| r}
$$

It may be noticed that (5.1) can be motivated as follows. Let us expand a particular function

$$
\frac{1}{t-z}, \quad|t|=1
$$

formally as in (4.1), by interpolation in the points $\beta_{n}$. The reader will verify the formula

$$
\frac{1}{t-z}-r_{n}(z)=\frac{\left(z-\beta_{1}\right) \cdots\left(z-\beta_{n}\right)\left(1-\bar{\beta}_{1} t\right) \cdots\left(1-\bar{\beta}_{n} t\right)}{\left(1-\bar{\beta}_{1} z\right) \cdots\left(1-\bar{\beta}_{n} z\right)(t-z)\left(t-\beta_{1}\right) \cdots\left(t-\beta_{n}\right)}
$$

By virtue of the equation $|t|=1$, the modulus of the right-hand member is

$$
\frac{1}{|t-z|}\left|\frac{\left(z-\beta_{1}\right) \cdots\left(z-\beta_{n}\right)}{\left(1-\bar{\beta}_{1} z\right) \cdots\left(1-\bar{\beta}_{n} z\right)}\right|
$$

which by (5.2) approaches zero for $|z|<1$. If the equation (5.4) is multiplied through by $f_{1}(t) d t$ and integrated over $C$, we have precisely (5.1). The righthand member of (5.4) approaches zero uniformly for $|z| \leqq r<1$, which implies the approach to zero of the right-hand member of (5.1), uniformly for $|z| \leqq r<1$.

Theorem III, in a somewhat less complete form and without the content of Theorem II, was proved by Malmquist (loc. cit.) for the case that $f(z)$ is of class $E_{2}$ and that the $\beta_{n}$ are all distinct and different from zero.

It is obvious that a function $f(z)$ of class $E$ which vanishes in the points $\beta_{n}$ must vanish identically interior to $C$, for its formal expansion (4.1) vanishes identically yet converges interior to $C$ to the function $f(z)$. 
THEOREM IV. If the product $\Pi\left|\beta_{n}\right|$ diverges, a necessary and sufficient condition that a function $f(z)$ of class $E$ vanish identically interior to $C$ is that the formal expansion (4.1) found either by interpolation in the points $\beta_{n}$ or by expanding $f_{1}(z)$ formally on $C$, should vanish identically.

Theorems III and IV have obvious significance relative to the problem of interpolation mentioned in the introduction. If the product $\prod\left|\beta_{n}\right|$ diverges and if a function of class $E$ exists which takes on the values $\gamma_{n}$ in the points $\beta_{n}$, then this function (required to be of class $E$ ) is unique and is represented by its formal development (4.1) found by interpolation in the points $\beta_{n}$.

6. Discussion of Theorem III. We shall now give illustrations to show the necessity of some restrictions on the points $\beta_{n}$ and on the function $f(z)$ for the validity of the development (4.1), to indicate that the hypothesis of Theorem III is not entirely accidental.

If the product $\Pi\left|\beta_{n}\right|$ is convergent, the conclusion of Theorem III certainly is not valid without restriction. Indeed, the product (3.1) itself is convergent and represents a function limited for $|z|<1$; the boundary values of this function $f(z)$ exist almost everywhere on $C$, by Fatou's theorem, and if we define $f_{1}(z)$ on $C$ as equal to these boundary values, equation (4.4) is valid. Yet $f(z)$ vanishes in each point $\beta_{n}$, so in its formal development (4.1) each coefficient is zero.

It may still occur, if the product $\prod\left|\beta_{n}\right|$ diverges, that the formal expansion (4.1) of a function $f(z)$ analytic for $|z|<1$ but not of class $E$ converges to $f(z)$ for $|z|<1$. This is necessarily the case if the points $\beta_{n}$ satisfy an inequality of the form $\left|\beta_{n}\right| \leqq \rho<1$, for an arbitrary function analytic for $|z|<1$, as we prove in $\$ 11$. Conceivably in other cases, if $f(z)$ is analytic for $|z|<1$ but $|f(z)|$ becomes infinite sufficiently slowly as $|z|$ approaches unity, and if the product $\prod\left|\beta_{n}\right|$ diverges sufficiently rapidly, the integral in (5.1) may be taken not over $C$ but over a variable circle $C_{n}:|z|=r_{n}<1$, containing the points $\beta_{1}, \beta_{2}, \cdots, \beta_{n}$ in its interior, and it may still be possible to prove convergence of the sequence $r_{n}(z)$ to $f(z)$ for $|z|<1$.

Indeed, we have seen that the function $f(z)=1 /(t-z),|t|=1$, is represented for $|z|<1$ by its formal development; yet this function is not of class $E$, for in its Maclaurin expansion (compare $\$ 2$ ) the $n$th coefficient does not approach zero with $1 / n$.

Let us give an example of a divergent infinite product $\Pi\left|\beta_{n}\right|$ and of a function $f(z)$ analytic for $|z|<1$ but not of class $E$, where the formal development (4.1) diverges. It follows directly from the definition of class $E$ that in (4.4) the function $f(z)$ satisfies an inequality

$$
(1-|z|)|f(z)| \leqq \frac{1}{2 \pi} \int_{C}\left|f_{1}(t)\right||d t|=M^{\prime},|z|<1 .
$$


Our present example consists of $f(z)=1 /(1-z)^{2}$, obviously not of class $E$. Let us set

$$
\frac{1}{(1-z)^{2}}-r_{n}(z)=M \frac{\left(z-\beta_{1}\right) \cdots\left(z-\beta_{n}\right)(z-\beta)}{\left(1-\bar{\beta}_{1} z\right) \cdots\left(1-\bar{\beta}_{n} z\right)(1-z)^{2}}
$$

where $M$ and $\beta$ are still to be determined. The function $r_{n}(z)$ here defined will be of the proper form (4.7) provided the function

$\phi(z)=\left(1-\bar{\beta}_{1} z\right)\left(1-\bar{\beta}_{2} z\right) \cdots\left(1-\bar{\beta}_{n} z\right)-M\left(z-\beta_{1}\right)\left(z-\beta_{2}\right) \cdots\left(z-\beta_{n}\right)(z-\beta)$

is divisible by $(1-z)^{2}$. This divisibility condition can be expressed by $\phi(1)=0, \phi^{\prime}(1)=0$. For the particular values (which we now choose)

$$
\beta_{1}=\frac{1}{2}, \quad \beta_{2}=\frac{2}{3}, \ldots, \beta_{n}=\frac{n}{n+1}
$$

one finds readily

$$
\beta=1+\frac{1}{n(n+2)}, \quad M=\frac{1}{1-\beta}=-n(n+2) .
$$

Hence for $z=0$ the right-hand member of $(6.1)$ becomes

$$
(-1)^{n+2} \frac{n(n+2)}{n+1}\left(1+\frac{1}{n(n+2)}\right) \text {, }
$$

which becomes infinite with $n$.

\section{THE PRODUCT $\prod\left|\beta_{n}\right|$ CONVERGENT}

7. Convergence of formal development. It is obvious that in case the product $\prod\left|\beta_{n}\right|$ converges, our previous proof of the convergence to $f(z)$ of the formal development (4.1) of the function $f(z)$ fails. Indeed, as we have pointed out in $\$ 6$, it is clear that not only our previous proofs but also the conclusions must be substantially modified if the product $\prod\left|\beta_{n}\right|$ converges. The following theorem is complementary to Theorem III.

THEOREM V. If the product $\Pi\left|\beta_{n}\right|$ converges and if $f(z)$ is of class $E$, then the formal development (4.1) of $f(z)$ (found either by interpolation in the points $\beta_{n}$ or by expansion of $f_{1}(z)$ on $C$ ) converges to the limit

$$
F(z)=f(z)-\frac{B(z)}{2 \pi i} \int_{C} \frac{f_{1}(t) d t}{(t-z) B(t)}
$$

for $|z|<1$, uniformly for $|z| \leqq r<1$. 
In the present case we study equation (5.1), now written in the equivalent form (notation of $\$ 3$ )

$$
f(z)-r_{n}(z)=\frac{B_{n}(z)}{2 \pi i} \int_{C} \frac{f_{1}(t) d t}{(t-z) B_{n}(t)} ;
$$

some modification is necessary in (3.3) and hence in (7.2) if the restriction $\beta_{n} \neq 0$ is not made, but the reader can either make the requisite modification or refer to our discussion in $\$ 9$. We have already shown (Theorem I) that $B_{n}(z)$ converges in the mean on $C$ to the function $B(z)$. By virtue of the fact that $B_{n}(z)$ is of constant modulus $\left|\beta_{1} \beta_{2} \cdots \beta_{n}\right|$ on $C$ and that $B(z)$ is of constant modulus $\prod\left|\beta_{n}\right|$ almost everywhere on $C$, ${ }^{*}$ it follows that $1 / B_{n}(z)$ converges in the mean on $C$ to the function $1 / B(z)$ :

$$
\begin{aligned}
\int_{C}\left|\frac{1}{B_{n}(z)}-\frac{1}{B(z)}\right|^{2}|d z| & =\int_{C}\left|\frac{B(z)-B_{n}(z)}{B_{n}(z) B(z)}\right|^{2}|d z| \\
& =\frac{1}{\left|\beta_{1} \beta_{2} \cdots \beta_{n}\right|{ }^{2} \prod\left|\beta_{n}\right|^{2}} \int_{C}\left|B(z)-B_{n}(z)\right|^{2}|d z| ;
\end{aligned}
$$

this last integral approaches zero. The proof of Theorem $\mathrm{V}$ is now immediate, if we notice that

$$
\lim _{n \rightarrow \infty} \int_{C} \frac{f_{1}(t) d t}{(t-z) B_{n}(t)}=\int_{C} \frac{f_{1}(t) d t}{(t-z) B(t)}
$$

by the lemma of $\$ 3$; the uniformity of the convergence for $|z| \leqq r<1$ is a consequence of the remark following that lemma. The uniformity of the convergence for $|z| \leqq r<1$ of $B_{n}(z)$ to $B(z)$ is also needed in this proof.

The function

$$
\frac{B(z)}{2 \pi i} \int_{C} \frac{f_{1}(t) d t}{(t-z) B(t)},|z|<1,
$$

which appears.in (7.1) obviously vanishes in the points $\beta_{n}$, so that $F(z)$ and $f(z)$ coincide in the points $\beta_{n}$. This must naturally be true, for any series (4.1) formed by interpolation to $f(z)$ in the points $\beta_{n}$ converges to the value $f(z)$ in those points $\beta_{n}$. The function $F(z)$ has the same formal development (4.1) as does $f(z)$. In particular if we set $f(z) \equiv B(z)$, we have $F(z) \equiv 0$.

THEOREM VI. If $f(z)$ is a function of class $E$, then whether or not the product $\Pi\left|\beta_{n}\right|$ diverges, the formal development (4.1) of $f(z)$ found either by interpolation to $f(z)$ in the points $\beta_{n}$ or by expansion of $f_{1}(z)$ on $C$, converges for $|z|<1$, uni-

* F. Riesz, Mathematische Zeitschrift, vol. 18 (1923), pp. 87-95.

Another proof of this remark is readily given by use of Theorem $I$. The function $B_{n}(z)$ approaches $B(z)$ in the mean on $C$, hence $\left|B_{n}(z)\right|$ (which has the constant value $\left|\beta_{1} \beta_{2} \cdots \beta_{n}\right|$ ) approaches $|B(z)|$ in the mean on $C$. Therefore $|B(z)|$ has the constant value $\prod\left|\beta_{n}\right|$ almost everywhere on $C$. 
formly for $|z| \leqq r<1$, to a function analytic for $|z|<1$ which coincides with $f(z)$ in the points $\beta_{n}$.

Theorem VI is of particular interest in connection with the problem of interpolation mentioned in the introduction. It informs us that whether or not the product $\Pi\left|\beta_{n}\right|$ diverges, if a function $f(z)$ of class $E$ exists which takes on the given values in the given points, the formal expansion (4.1) converges for $|z|<1$, uniformly for $|z| \leqq r<1$.

If the product $\prod\left|\beta_{n}\right|$ converges, a necessary and sufficient condition that the function $f(z)$ of class $E$ be represented by its formal expansion (4.1), is the equation

$$
\int_{C} \frac{f_{1}(t) d t}{(t-z) B(t)} \equiv 0,|z|<1,
$$

and this condition (compare $\$ 2$ ) can be written

$$
\int_{c} \frac{f_{1}(t) d t}{B(t) t^{n}}=0 \quad(n=1,2,3, \cdots) .
$$

It may be noticed that the method of proof of Theorem $\mathrm{V}$ can be interpreted as was the proof of Theorem III. We expand, namely, the function $1 /(t-z),|t|=1$, in a series of form (4.1), by interpolation in the points $\beta_{n}$. This series is found to converge to the function

$$
\frac{1}{t-z}-\frac{B(z)}{(t-z) B(t)}
$$

for $|z|<1$, uniformly for $|z| \leqq r<1$. Multiplication of the series term by term by $f_{1}(t) d t$ and integration over $C$ then yields a proof of the convergence of the formal development of the function $f(z)$, together with a formula for the sum of the series.

Let us give an example, similar to the one of $\S 6$, to show that the formal expansion of an arbitrary function $f(z)$ analytic for $|z|<1$ need not converge for $|z|<1$. We choose

$$
f(z)=\frac{1}{(1-z)^{2}}, \quad \beta_{n}=1-\frac{1}{2^{n}} ;
$$

the product $\prod\left|\beta_{n}\right|$ converges. We consider equation (6.1) to hold, where $M$ and $\beta$ are still to be determined. The function

$$
\begin{aligned}
\phi(z)=\left(1-\bar{\beta}_{1} z\right)\left(1-\bar{\beta}_{2} z\right) \cdots\left(1-\bar{\beta}_{n} z\right) & \\
\quad & \quad M\left(z-\beta_{1}\right)\left(z-\beta_{2}\right) \cdots\left(z-\beta_{n}\right)(z-\beta)
\end{aligned}
$$


is divisible by $(1-z)^{2}$. This divisibility condition can be expressed by $\phi(1)=0$, $\phi^{\prime}(1)=0$. For the values $\beta_{n}$ already indicated we find readily

$$
\beta=\frac{2^{n+1}}{2^{n+1}-1}, \quad M=\frac{1}{1-\beta}=1-2^{n+1} .
$$

Then for the particular value $z=0$ the right-hand member of (6.1) becomes

$$
1-r_{n}(0)=(-1)^{n-1} M \beta_{1} \beta_{2} \cdots \beta_{n}(-\beta)=(-1)^{n} \beta_{1} \beta_{2} \cdots \beta_{n}\left(2^{n+1}\right),
$$

which becomes infinite with $n$.

8. Functions of class $E_{2}$. The situation of Theorem V is particularly interesting if $f(z)$ is of class $E_{2}$ and the product $\prod\left|\beta_{n}\right|$ converges. The present sequence of functions (4.3) in terms of which $f_{1}(z)$ is developed formally on $C$ does not form a complete set of orthogonal functions on $C$ with respect to functions of class $E_{2}$. In the present case, this means that there exist functions $f_{1}(z)$ (for instance $B(z)$ ) integrable together with $\left[f_{1}(z)\right]^{2}$ on $C$, orthogonal on $C$ to all of the functions (4.3), yet such that

$$
f(z)=\frac{1}{2 \pi i} \int_{C} \frac{f_{1}(t) d t}{t-z} .
$$

is not identically zero interior to $C$; the formal development (4.1) of such a function vanishes identically. In a sense, the completeness of the set (4.3) is the essential difference between the case $\Pi\left|\beta_{n}\right|$ divergent and the case $\prod\left|\beta_{n}\right|$ convergent. If the product $\prod\left|\beta_{n}\right|$ diverges, there is no function $f(z)$ even of class $E$ not identically zero interior to $C$ whose formal development (4.1) vanishes identically, for the formal development (4.1) in this case always converges interior to $C$ to the function $f(z)$ itself.

THEOREM VII. If $f(z)$ is of class $E_{2}$ :

$$
f(z)=\frac{1}{2 \pi i} \int_{C} \frac{f_{1}(t) d t}{t-z},|z|<1,
$$

where $f_{1}(t)$ is integrable together with its square on $C$, and if the product $\prod\left|\beta_{n}\right|$ converges, then the formal expansion of $f(z)$ in a series (4.1) found either by expanding $f_{1}(z)$ on $C$ or by interpolation to $f(z)$ in the points $\beta_{n}$, converges in the mean on $C$ to some function $f_{2}(z)$ integrable and with an integrable square on $C$. This series (4.1) converges for $|z|<1$, uniformly for $|z| \leqq r<1$, to the function of class $E_{2}$

$$
F(z)=\frac{1}{2 \pi i} \int_{C} \frac{f_{2}(t) d t}{t-z},|z|<1 .
$$


The function $f_{2}(t)$ (defined almost everywhere on $C$ ) is characterized as the (essentially unique) function of minimum norm $\int_{C}\left|f_{2}(t)\right|^{2}|d t|$ whose formal development on $C$ is the formal development (4.1) of $f_{1}(t)$. Moreover, the function $f_{2}(t)$ is the boundary value of $F(t)$ taken on almost everywhere on $C$ in the sense of radial approach, and the equation

is valid.

$$
\frac{1}{2 \pi i} \int_{C} \frac{f_{2}(t) d t}{t-z}=0,|z|>1,
$$

It follows from the Riesz-Fischer theorem that the formal expansion (4.1) of $f_{1}(z)$ on $C$ converges in the mean on $C$ to some function $f_{2}(z)$ integrable and with an integrable square on $C$. Any other function $f_{3}(z)$ integrable and with an integrable square on $C$ which has the formal expansion (4.1) is such that $f_{3}(z)-f_{2}(z)$ is orthogonal to each of the functions (4.3) and hence is orthogonal to $f_{2}(z)$ on $C$ :

$$
\begin{aligned}
\int_{C}\left[f_{3}(z)-f_{2}(z)\right] \bar{f}_{2}(z)|d z| & \\
& =\int_{C}\left[\bar{f}_{3}(z)-\bar{f}_{2}(z)\right] f_{2}(z)|d z|=0 .
\end{aligned}
$$

A necessary and sufficient condition that $f_{2}(z)$ and $f_{3}(z)$ be essentially distinct, that is, different from each other on a set of points on $C$ of positive measure, is the inequality

$$
0<\int_{C}\left[f_{3}(z)-f_{2}(z)\right]\left[\bar{f}_{3}(z)-\bar{f}_{2}(z)\right]|d z|
$$

and this right-hand member by virtue of (8.2) reduces to

$$
\int_{C} f_{3}(z) \bar{f}_{3}(z)|d z|-\int_{C} f_{2}(z) \bar{f}_{2}(z)|d z| .
$$

Then the norm of $f_{3}(z)$ on $C$ is greater than the norm of $f_{2}(z)$ on $C$. That is to say, $f_{2}(z)$ is the unique function integrable and with an integrable square on $C$ of minimum norm whose formal development on $C$ is (4.1), the formal development on $C$ of $f_{1}(z)$. The norm of $f_{2}(z)$ on $C$ is by Parseval's theorem

$$
2 \pi \sum \frac{\left|a_{n-1}\right|^{2}}{1-\left|\beta_{n}\right|^{2}} \text {. }
$$

The various terms of the series (4.1) are analytic on and within $C$, so the convergence of (4.1) interior to $C$ to the function $F(z)$ follows directly from the 
LEMma. If the sequence of functions $\psi_{n}(z)$ each integrable on $C$ converges in the mean on $C$ to the function $\psi(z)$, then the sequence

$$
\frac{1}{2 \pi i} \int_{C} \frac{\psi_{n}(t) d t}{t-z},|z| \neq 1,
$$

converges, uniformly for $|z| \leqq r<1$ or $|z| \geqq R>1$, to the function

$$
\frac{1}{2 \pi i} \int_{C} \frac{\psi(t) d t}{t-z} .
$$

This lemma (for $|z|<1$ ) is used by Malmquist (loc. cit.) and can be proved easily from the inequality of Schwarz. We omit the proof. The lemma is readily extended to apply to an arbitrary rectifiable curve $C$, and to $z$ either interior or exterior to $C$.

The only remaining part of Theorem VII to be proved is that $f_{2}(z)$ is the boundary value of $F(z)$ taken on almost everywhere on $C$ for radial approach. Since each term $a_{n} \phi_{n}(z)$ of the series (4.1) is analytic on and within $C$, we have

$$
\frac{1}{2 \pi i} \int_{C} \frac{a_{n} \phi_{n}(t) d t}{t-z}=0,|z|>1 .
$$

Then by the lemma we have

$$
\frac{1}{2 \pi i} \int_{C} \frac{f_{2}(t) d t}{t-z}=0,|z|>1 .
$$

Our conclusion, that $f_{2}(z)$ is the boundary value of $F(z)$ taken on almost everywhere on $C$ for radial approach, now follows from a theorem due to $\mathrm{F}$. and $\mathrm{M}$. Riesz (loc. cit.).

It is worth noticing that the function

$$
f(z)-F(z)
$$

is of class $E_{2}$ (the difference of two functions of class $E_{2}$ ) and vanishes in the points $\beta_{n}$. It has been indicated in $\S 2$ that for an arbitrary function $\phi(z)$ of class $E_{2}$, boundary values $\phi(z)$ exist almost everywhere on $C$ in the sense of radial approach; the function $\phi(z)$ is integrable together with its square on $C$, and we have

$$
\phi(z)=\frac{1}{2 \pi i} \int_{C} \frac{\phi(t) d t}{t-z},|z|<1 .
$$

Since $B(z)$ has a constant modulus almost everywhere on $C$, these values being defined almost everywhere on $C$ by radial approach, the function 


$$
\theta(z)=\frac{f(z)-F(z)}{B(z)}
$$

also has boundary values $\theta(z)$ almost everywhere on $C$ in the sense of radial approach, and we have

$$
\theta(z)=\frac{1}{2 \pi i} \int_{C} \frac{\theta(t) d t}{t-z},
$$

where $\theta(z)$ is integrable together with its square on $C$. That is to say, the function $F(z)$ can be written in the form

where $\theta(z)$ is of class $E_{2}$.

$$
F(z)=f(z)-B(z) \theta(z),
$$

Another view of this equation is to consider $F(z)$ as given:

$$
f(z)=F(z)+B(z) \theta(z) .
$$

That is, let $F(z)$ be defined from a given formal development (4.1) and satisfy the conditions of $F(z)$ in Theorem VII. Then any function $f(z)$ of class $E_{2}$ which has this formal development (4.1) can be expressed by (8.3), where $\theta(z)$ is a suitable function of class $E_{2}$. Reciprocally, any function $f(z)$ which can be given by (8.3), where $\theta(z)$ is of class $E_{2}$, is also of class $E_{2}$ and has this given formal development (4.1). The formal development (4.1) of $f(z)$ may naturally be found either by expansion on $C$ or by interpolation in the points $\beta_{n}$.

Thus, a necessary and sufficient condition that two functions $f(z)$ and $g(z)$ of class $E_{2}$ have the same development (4.1) (found either by expansion on $C$ or by interpolation in the points $\left.\beta_{n}\right)$ is that $f(z)-g(z)$ can be written $B(z) \phi(z)$, where $\phi(z)$ is a function of class $E_{2}$.

If the product $\prod\left|\beta_{n}\right|$ diverges, it is still true (compare Malmquist, loc. cit.) that the formal development (4.1) of the function $f(z)$ of class $E_{2}$, found either by expanding $f_{1}(z)$ formally on $C$ or by interpolation to $f(z)$ in the points $\beta_{n}$, converges in the mean on $C$ to some function $f_{2}(z)$. The two functions $f_{1}(z)$ and $f_{2}(z)$ need not be equal almost everywhere on $C$, but (a) if $f_{1}(z)$ is known to be the boundary values of $f(z)$ almost everywhere on $C$ in the sense of radial approach, or (b) if $f_{1}(z)$ is known to be the function of minimum norm such that

$$
f(z)=\frac{1}{2 \pi i} \int_{C} \frac{f_{1}(t) d t}{t-z},|z|<1,
$$

is valid, or (c) if the equation

$$
\frac{1}{2 \pi i} \int_{C} \frac{f_{1}(t) d t}{t-z}=0,|z|>1,
$$

is known to hold, then $f_{1}(z)$ and $f_{2}(z)$ are equal almost everywhere on $C$. 
The functions (4.3) are not in their present form normal on $C$ (i.e. $\left.\int_{C}\left|\phi_{n}(z)\right|^{2}|d z| \neq 1\right)$. We find

$$
\int_{C}\left|\phi_{n-1}(z)\right|^{2}|d z|=\frac{2 \pi}{1-\beta_{n} \bar{\beta}_{n}}
$$

Then by the Riesz-Fischer theorem a necessary and sufficient condition that (4.1) be the formal development on $C$ of a function integrable and with an integrable square on $C$ is that the series

$$
2 \pi \sum \frac{\left|a_{n-1}\right|^{2}}{1-\beta_{n} \bar{\beta}_{n}} \text { or } \sum \frac{\left|a_{n-1}\right|^{2}}{1-\left|\beta_{n}\right|}
$$

converge.

In connection with the problem on interpolation mentioned in the introduction we may now state our main theorem:

THEOREM VIII. The following conditions are all equivalent:

(1) that the series (4.1) converge in the mean on $C$;

(2) that the series

$$
\sum \frac{\left|a_{n-1}\right|^{2}}{1-\left|\beta_{n}\right|}
$$

converge;

(3) that the series (4.1) be the formal expansion on $C$ of a function $f_{1}(z)$ integrable and with an integrable square on $C$;

(4) that a function $f(z)$ of class $E_{2}$ exist of which (4.1) is the formal development found by interpolation in the points $\beta_{n}$;

(5) that the series (4.1) converge for $|z|<1$ to a function $F(z)$ of class $E_{2}$.

These conditions (1)-(5) are all equivalent whether $\left|\beta_{n}\right|$ converges or diverges, and the proof has already been given in every case.

If $f(z)$ is of class $E_{2}$, and if $\coprod\left|\beta_{n}\right|$ converges, the formal expansion (4.1) of $f(z)$ found either by interpolation in the points $\beta_{n}$ or by expansion of $f_{1}(z)$ on $C$, is such that $\sum\left|a_{n}\right|$ converges, as we now proceed to prove. Series $\sum\left(1-\left|\beta_{n}\right|\right)$ converges, by the convergence of the infinite product. It is well known that the series $\sum\left|A_{n} B_{n}\right|$ converges provided the series $\sum\left|A_{n}\right|^{2}$ and $\sum\left|B_{n}\right|^{2}$ converge. The convergence of (8.4) and of $\sum^{n}\left(1-\left|\beta_{n}\right|\right)$ implies then the convergence of $\sum\left|a_{n}\right|$. It follows directly from (4.1), if we assume now that $\sum\left|a_{n}\right|$ converges, that the series (4.1) converges uniformly on any closed point set for which $|z| \leqq 1$ and which contains no limit point of the $\beta_{n}$. This gives an immediate proof that $f_{2}(z)$ is the boundary value of $F(z)$ under certain restrictions on the sequence $\beta_{n}$ (for instance if the points $\beta_{n}$ have only a finite number of limit points on $C$ ) and allows approach to points of $C$ more 
general than radial approach or even more general than approach to points of $C$ in a triangle whose interior is interior to $C$.

Part of this discussion, if not all, given in $\S 8$ can be extended from functions of class $E_{2}$ to functions of class $E_{p}, p>1$. $^{*}$ For instance, it is seen by inspection of (7.1) that if $f(z)$ is of class $E_{p}$, then we have

$$
F(z)=f(z)-B(z) \theta(z),
$$

where $\theta(z)$ is of class $E_{p}$. For $B(t)$ is of constant modulus almost everywhere on $C$, so if $f_{1}(t)$ is integrable on $C$ together with its $p$ th power, so also is $f_{1}(t) / B(t)$. In any case it is clear that if $f(z)$ belongs to $E$, then $F(z)-f(z)$ is of the form $B(z) \theta(z)$, where $\theta(z)$ is of class $E$. Likewise if $f(z)$ is of class $E^{\prime}$, then $F(z)-f(z)$ is of the form $B(z) \theta(z)$, where $\theta(z)$ is of class $E^{\prime}$.

If $f(z)$ is an arbitrary function of class $E$,

$$
f(z)=\frac{1}{2 \pi i} \int_{C} \frac{f_{1}(t) d t}{t-z},|z|<1,
$$

where $f_{1}(t)$ is integrable on $C$, and if $f(z)$ itself vanishes in all the points $\beta_{n}$, then $F(z)$ vanishes identically and we have

$$
f(z)=\frac{B(z)}{2 \pi i} \int_{C} \frac{f_{1}(t) d t}{(t-z) B(t)},|z|<1,
$$

a general representation for such functions. If $f(z)$ is not merely of class $E$ but also of class $E_{p}$, the integral in (8.5) is also a function of class $E_{p}$; our result in this case is similar to a result due to F. Riesz. $\dagger$

In the case $p=2$, the converse is easy to prove. Let us suppose

$$
f(z)=\frac{B(z)}{2 \pi i} \int_{C} \frac{f_{1}(t) d t}{t-z},|z|<1,
$$

where $f_{1}(t)$ is integrable together with its square on $C$; we are to prove that $f(z)$ is of class $E_{2}$. We set

$$
\theta(z)=\frac{1}{2 \pi i} \int_{C} \frac{f_{1}(t) d t}{t-z}=a_{0}+a_{1} z+a_{2} z^{2}+\cdots,|z|<1,
$$

and the convergence of the series $\sum\left|a_{n}\right|^{2}$ is assured. We have

$$
\frac{1}{2 \pi r} \int_{|z|=r}|\theta(z)|^{2}|d z|=\left|a_{0}\right|+\left|a_{1}\right| r+\left|a_{2}\right| r^{2}+\cdots, r<1,
$$

* See particularly F. Riesz, Mathematische Zeitschrift, vol. 18 (1923), pp. 117-124.

$\dagger$ Mathematische Zeitschrift, vol. 18 (1923), pp. 87-95. 
so the left-hand member is uniformly limited for all $r<1$. But $B(z)$ is uniformly limited for $|z|<1$ and hence

$$
\frac{1}{2 \pi r} \int_{|z|=r}|\theta(z) B(z)|^{2}|d z|
$$

is uniformly limited for all $r<1$, whence it follows (F. and M. Riesz, loc. cit.) that $f(z)$ is of class $E_{2}$.

9. Case $\beta_{1}=0$. Practically all of the formulas we have hitherto used in the present paper are valid, with perhaps obvious alterations in such formulas as (3.1) and $\prod\left|\beta_{n}\right|$, even if we have $\beta_{1}=0$ or more generally if several of the numbers $\beta_{n}$ are equal to zero.

In the previous work by the present writer (loc. cit.), it was primarily a question of series of form (1.1). The natural procedure for the present study would, by analogy, be to orthogonalize the sequence

$$
1, \frac{1}{z-\alpha_{1}}, \frac{1}{z-\alpha_{2}}, \ldots,\left|\alpha_{n}\right|>1
$$

on $C$. This leads directly to the functions

$$
1 ; \frac{z}{z-\alpha_{1}}, \frac{z\left(1-\bar{\alpha}_{1} z\right)}{\left(z-\alpha_{1}\right)\left(z-\alpha_{2}\right)}, \frac{z\left(1-\bar{\alpha}_{1} z\right)\left(1-\bar{\alpha}_{2} z\right)}{\left(z-\alpha_{1}\right)\left(z-\alpha_{2}\right)\left(z-\alpha_{3}\right)}, \ldots,
$$

which lead necessarily to a series

$$
a_{0}+a_{1} \frac{z}{z-\alpha_{1}}+a_{2} \frac{z\left(1-\bar{\alpha}_{1} z\right)}{\left(z-\alpha_{1}\right)\left(z-\alpha_{2}\right)}+\cdots
$$

of form (1.1), involving interpolation in the origin. On the other hand (compare Malmquist, loc. cit.), one can orthogonalize the sequence

$$
\frac{1}{z-\alpha_{1}}, \frac{1}{z-\alpha_{2}}, \frac{1}{z-\alpha_{3}}, \ldots,\left|\alpha_{n}\right|>1,
$$

on $C$. This yields the set

$$
\frac{1}{z-\alpha_{1}}, \frac{1-\bar{\alpha}_{1} z}{\left(z-\alpha_{1}\right)\left(z-\alpha_{2}\right)}, \frac{\left(1-\bar{\alpha}_{1} z\right)\left(1-\bar{\alpha}_{2} z\right)}{\left(z-\alpha_{1}\right)\left(z-\alpha_{2}\right)\left(z-\alpha_{3}\right)}, \ldots
$$

which leads necessarily to a series of form

(9.6) $a_{1} \frac{1}{z-\alpha_{1}}+a_{2} \frac{1-\bar{\alpha}_{1} z}{\left(z-\alpha_{1}\right)\left(z-\alpha_{2}\right)}+a_{3} \frac{\left(1-\bar{\alpha}_{1} z\right)\left(1-\bar{\alpha}_{2} z\right)}{\left(z-\alpha_{1}\right)\left(z-\alpha_{2}\right)\left(z-\alpha_{3}\right)}+\cdots$, 
which completely excludes interpolation in the origin. To be sure, one may interpret $1 /\left(z-\alpha_{1}\right)$ in (9.4) for $1 / \alpha_{1}=0$ as the function $z$ itself, but then (9.6) becomes

$$
a_{1} z+a_{2} \frac{z^{2}-\alpha_{2} z+\alpha_{2}^{2}}{z-\alpha_{2}}+\cdots
$$

which is not a series whose coefficients are readily found by interpolation.

For purposes of the present paper, it is much more advantageous to commence with the set

$$
\frac{1}{1-\bar{\beta}_{1} z}, \frac{1}{1-\bar{\beta}_{2} z}, \frac{1}{1-\bar{\beta}_{3} z}, \ldots,\left|\beta_{n}\right|<1,
$$

instead of (9.1) or (9.4). By othogonalization we obtain precisely (4.3). The set (9.7) has essentially both (9.1) and (9.4) as special cases.

All of the sets (9.1), (9.4), (9.7) are to be modified if the $\alpha_{n}$ (or $\beta_{n}$ ) are not all distinct, provided the corresponding series are to be used for interpolation with the convention we have introduced. For instance, if in (9.1) or (9.4) the function $1 /(z-\alpha)$ appears $k$ times, this sequence of $1 /(z-\alpha)$ repeated is to be replaced by

$$
\frac{1}{z-\alpha}, \frac{1}{(z-\alpha)^{2}}, \cdots, \frac{1}{(z-\alpha)^{k}}
$$

In (9.7) a function $1 /(1-\bar{\beta} z)$ occurring $k$ times is to be treated similarly, except that in case $\beta=0$ we use instead the sequence $1, z, z^{2}, \cdots, z^{k-1}$. In these respective cases, the formulas (9.2), (9.5), (4.3) are all valid in their present forms.

In connection with Theorem II and the remark following it, the following theorem (a consequence of that remark) has some interest:

If $f_{1}(z)$ is integrable on $C$ together with its square and if we have

then the function

$$
f(z)=\frac{1}{2 \pi i} \int_{C} \frac{f_{1}(t) d t}{t-z},|z|<1,
$$

$$
\frac{a_{0 n} z^{n}+a_{1 n} z^{n-1}+\cdots+a_{n n}}{\left(1-\bar{\beta}_{1} z\right)\left(1-\bar{\beta}_{2} z\right) \cdots\left(1-\bar{\beta}_{n} z\right)}
$$

of best approximation to $f_{1}(z)$ on $C$ in the sense of least squares, where the $\beta_{k}$ are given, is the unique function of form (9.8) which coincides with $f(z)$ in the points $0, \beta_{1}, \beta_{2}, \cdots, \beta_{n}$.

The function (9.8) is the most general function formally of degree $n$ with its poles in the points $\beta_{k}$, whereas the function (4.7) automatically vanishes (formally) at infinity. If such rational functions are to be studied under linear 
transformation of the complex variable, the natural form to use is (9.8) instead of (4.7) (compare Walsh, loc. cit.), although to be sure (4.7) gives us essentially (9.8) with $n$ replaced by $n-1$, if we set $\beta_{1}=0$. The condition that a function (4.7) vanish at infinity is clearly not invariant under linear transformation.

We see, then, that the case $\beta_{1}=0$ (or any $\beta_{k}=0$ ) is not exceptional for the treatment we have given, except that in certain formulas for products such as $\prod\left|\beta_{n}\right|$ a slight formal modification may have to be made. In spite of the inclusiveness of our method, it is instructive to notice the relation between the case where the restriction $\beta_{k} \neq 0$ is made and the case where it is not made. We add two remarks (i) and (ii) on the relations between these two cases.

(i) The cases $\beta_{n} \neq 0$ and $\beta_{1}=0$ can be transformed each into the other by a transformation of the form

$$
w=\frac{z-\beta}{1-\bar{\beta} z}
$$

which leaves $C$ invariant. If a sequence $\beta_{n}$ is given not including the point $z=0$, a transformation (9.9) can be chosen which carries this sequence into a new sequence including the point $w=0$. Reciprocally, if a sequence $\beta_{n}$ is given which contains the point $z=0$, a transformation (9.9) can be chosen which transforms this sequence into a new sequence not containing the point $w=0$. In every case, the transformation (9.9) naturally transforms a sequence $\beta_{n}$ interior to $C$ into a sequence interior to $C$.

The convergence or divergence of the product $\Pi\left|\beta_{n}\right|$ is not affected by a transformation (9.9). Indeed, the convergence of such a product is a necessary and sufficient condition for the existence of a function analytic and limited interior to $C$ and vanishing at the points $\beta_{n}$. This existence is obviously invariant under a transformation (9.9).

Properties of orthogonality on $C$ and of best approximation on $C$ in the sense of least squares are naturally altered by a transformation (9.9), but if two functions $\phi(z)$ and $\psi(z)$ are orthogonal on $C$ :

$$
\int_{C} \phi(z) \bar{\psi}(z)|d z|=0,
$$

then we have

$$
\int_{C}\left|\frac{d z}{d w}\right| \phi(z) \bar{\psi}(z)|d w|=0,
$$

so $\phi(z)$ and $\psi(z)$ (both defined on $|w|=1$ by (9.9)) are orthogonal on $|w|=1$ with respect to the norm function $|d z / d w|$. Similarly, best approximation on 
$C:|z|=1$ in the sense of least squares corresponds to best approximation on $|w|=1$ in the sense of least squares with the norm function $|d z / d w|$.

The principal results which we have proved in the present paper relative to interpolation in the points $\beta_{n}$, where we have $\beta_{n} \neq 0$, are valid even without the restriction $\beta_{n} \neq 0$, as the reader can now prove by the use of (9.9), except insofar as those results apply to the specific form of series (4.3) or (9.3) as contrasted with (9.6).

Similarly, if we had started with (9.6) instead of (4.3), the results established for (9.6) could have been used directly in proving similar results for (4.3). It is a matter of taste whether (4.3) or (9.6) is taken as fundamental, but (4.3) is formally more inclusive.

(ii) The problem of expanding a function $f(z)$ analytic for $|z|<1$ in a series (9.6), $\left|\beta_{n}\right|<1$, is essentially the same as the problem of expanding the function

$$
\frac{f(z)-a_{0}}{z}, a_{0}=f(0)
$$

in a series of form (4.2). Thus, if we have $\beta_{1}=0, \beta_{1+k} \neq 0$, the problem of interpolation for $f(z)$ in the points $\beta_{n}$ is reduced to the problem of interpolation for (9.10) in the points $\beta_{1+k}$, a problem which we have already discussed in detail and solved in certain cases. To be sure, the function (9.10) has an artificial singularity at the origin, but here and below we consider such a singularity to be removed.

Even if a number of points $\beta_{n}$ coincide at $z=0$, a transformation of $f(z)$ similar to (9.10) can be made; if we assume $\beta_{1}, \beta_{2}, \cdots, \beta_{m}$ all zero but $\beta_{m+k} \neq 0$, then we may replace $(9.10)$ by

$$
\frac{f(z)-a_{0}-a_{1} z-\cdots-a_{m-1} z^{m-1}}{z^{m}},
$$

where these coefficients $a_{k}$ are found as in the expansion of $f(z)$ in Taylor's series.

As a general remark, it is clear that the expansion of $f(z)$ in a series of form (4.1) is equivalent to the expansion of the function $\left(1-\bar{\beta}_{1} z\right) f(z)$ in a series

$$
a_{0}+a_{1} \frac{z-\beta_{1}}{1-\bar{\beta}_{2} z}+a_{2} \frac{\left(z-\beta_{1}\right)\left(z-\beta_{2}\right)}{\left(1-\bar{\beta}_{2} z\right)\left(1-\bar{\beta}_{3} z\right)}+\cdots
$$

or is equivalent to the expansion of the function 


$$
\begin{gathered}
\frac{\left(1-\bar{\beta}_{1} z\right) \cdots\left(1-\bar{\beta}_{n} z\right)}{\left(z-\beta_{1}\right) \cdots\left(z-\beta_{n}\right)}\left[f(z)-a_{0} \frac{1}{1-\bar{\beta}_{1} z}-a_{1} \frac{z-\beta_{1}}{\left(1-\bar{\beta}_{1} z\right)\left(1-\bar{\beta}_{2} z\right)}-\cdots\right. \\
\left.\quad-a_{n-1} \frac{\left(z-\beta_{1}\right) \cdots\left(z-\beta_{n-1}\right)}{\left(1-\bar{\beta}_{1} z\right) \cdots\left(1-\bar{\beta}_{n} z\right)}\right]
\end{gathered}
$$

in a series of the form

$$
a_{n+1} \frac{1}{1-\bar{\beta}_{n+1} z}+a_{n+2} \frac{z-\beta_{n+1}}{\left(1-\bar{\beta}_{n+1} z\right)\left(1-\bar{\beta}_{n+2} z\right)}+\cdots
$$

This last series is again a series of form (4.1), and this remark can be used to generalize results already found for series (4.1); compare Walsh, loc. cit.

10. Interpolation at the origin. An aesthetic justification for the use of (9.3) rather than (9.6) is that (9.3) (and likewise (4.3)) includes Taylor's series as a special case, while (9.6) does not. The facts analogous to Theorems II-VIII are easily obtained for the case that all the $\beta_{n}$ are zero, and we state them because of their general interest. It will be noticed that in $\$ 9$ (i), the case that all the $\beta_{n}$ are zero is no exception, but in (ii) that case is an exception and cannot be handled by the method suggested, expansion of the function (9.11).

We proceed to state the results, then, for the case $\beta_{n}=0, n=1,2, \cdots$, special cases of results already proved. These results contain little that is new, ${ }^{*}$ but it is well to have them for comparison with the more general results we have proved for the series (4.1).

Let the function $f(z)$ be of class $E$, and represented by the equation

$$
f(z)=\frac{1}{2 \pi i} \int_{C} \frac{f_{1}(t) d t}{t-z},|z|<1,
$$

where $f_{1}(t)$ is integrable on $C$.

The formal expansion of $f(z)$ in Taylor's series

$$
f(z) \sim a_{0}+a_{1} z+a_{2} z^{2}+\cdots,
$$

found by interpolation in the origin, is identical with the formal expansion on $C$ of the function $f_{1}(z)$ in terms of the functions $1, z, z^{2}, \cdots$, orthogonal on $C$.

The polynomial of degree $n$ which is the best approximation to $f_{1}(z)$ on $C$ in the sense of least squares is the sum of the first $n+1$ terms of (10.2), provided the square of $f_{1}(z)$ is integrable on $C$.

Series (10.2) converges to the value $f(z)$ for $|z|<1$, uniformly for $|z| \leqq r<1$, and if $f(z)$ can be extended analytically for $|z|<R>1$, series (10.2) converges for $|z|<R$, uniformly for $|z| \leqq R^{\prime}<R$.

\footnotetext{
* F. and M. Riesz, loc. cit.; Walsh, these Transactions, vol. 32 (1930), pp. 335-390, \$12. 1.
} 
If $f_{1}(z)$ has an integrable square on $C$, the series $\sum\left|a_{n}\right|^{2}$ converges and series (10.2) converges in the mean on $C$ to some function $f_{2}(z)$ integrable and with an integrable square on $C$; the function $f(z)$ takes on the boundary values $f_{2}(z)$ for radial approach to $C$, almost everywhere on $C$. The following relations are valid:

$$
\begin{aligned}
& f(z)=\frac{1}{2 \pi i} \int_{C} \frac{f_{2}(t) d t}{t-z},|z|<1 ; \\
& \int_{C} \frac{f_{2}(t) d t}{t-z}=0, \cdot z \mid>1 ; \\
& \int_{C} f_{1}(t) t^{n} d t=\int_{C} f_{2}(t) t^{n} d t \quad(n=-1,-2,-3, \cdots) ; \\
& \int_{C} f_{2}(t) t^{n} d t=0 \quad(n=0,1,2, \cdots) .
\end{aligned}
$$

The relations (10.3) and (10.5) are equivalent, and so likewise are (10.4) and (10.6). Either of the relations (10.3) and (10.5) tagether with either of the relations (10.4) and (10.6) is sufficient to characterize $f_{2}(z)$ (integrable together with its square on $C$ ) completely. The formal expansion of $f_{2}(z)$ on $C$ in terms of the orthogonal functions $1, z, z^{2}, \cdots$ is precisely $(10.2)$, and $f_{2}(z)$ is the unique function of minimum norm on $C$ (namely $2 \pi \sum\left|a_{n}\right|^{2}$ ) which has the particular formal expansion (10.2) on $C$.

An arbitrary series (10.2), where the series $\sum\left|a_{n}\right|^{2}$ converges, is the formal expansion of some function $f(z)$ of class $E_{2}$, found either by interpolation to $f(z)$ in the origin, by formal expansion of the function $f_{1}(z)$ of $(10.1)$ on $C$, or by formal expansion of the function $f_{2}(z)$ on $C$; these three methods of engendering (10.2) are equivalent.

We have not yet studied the possibility of convergence exterior to $C$ of the general series (4.1); we turn shortly (in \$11) to the investigation of this possibility.

As a complement to the theorem stated and under its hypothesis, we state the obvious special case of Theorem IV:

$A$ necessary and sufficient condition that the function $f(z)$ represented by (10.1) should vanish identically interior to $C$ is that we have

or that we have

$$
f^{(n)}(0)=0 \quad(n=0,1,2, \cdots),
$$

that is,

$$
\int_{C} f_{1}(t) \bar{t}^{n}|d t|=0 \quad(n=0,1,2, \cdots),
$$

$$
\int_{C} \frac{f_{1}(t) d t}{t^{n}}=0 \quad(n=1,2, \cdots) .
$$




\section{SUPPLEMENTARY RESULTS}

11. Further results on convergence of (4.1); product $\prod\left|\beta_{n}\right|$ divergent. There are various results on the convergence of (4.1), supplementary to Theorems III and V, which should be mentioned; for instance cases of convergence for $|z|=1$ or $|z|>1$, and even cases of convergence at some points interior to $C$ when $f(z)$ is singular interior to $C$.

If the product $\prod\left|\beta_{n}\right|$ diverges and if $f(z)$ is analytic for $|z| \leqq 1$, then the formal expansion (4.1) converges to $f(z)$ uniformly for $|z| \leqq 1$.

We use again equation (5.1), except that the integral is now taken over a circle $C^{\prime}:|z|=T>1$ having on or within it no singularity of $f(z)$. Then the equation

$$
\begin{aligned}
f(z) & -r_{n}(z) \\
& =\frac{1}{2 \pi i} \int_{C^{\prime}} f(t) \frac{\left(z-\beta_{1}\right) \cdots\left(z-\beta_{n}\right)\left(1-\bar{\beta}_{1} t\right) \cdots\left(1-\bar{\beta}_{n} t\right)}{\left(1-\bar{\beta}_{1} z\right) \cdots\left(1-\bar{\beta}_{n} z\right)(t-z)\left(t-\beta_{1}\right) \cdots\left(t-\beta_{n}\right)} d t
\end{aligned}
$$

is valid for $|z| \leqq 1$. For $|z| \leqq 1$ we have

$$
\left|\frac{\left(z-\beta_{1}\right) \cdots\left(z-\beta_{n}\right)}{\left(1-\bar{\beta}_{1} z\right) \cdots\left(1-\bar{\beta}_{n} z\right)}\right| \leqq 1,
$$

and for $|t|=T$ the expression

$$
\frac{\left(1-\bar{\beta}_{1} t\right) \cdots\left(1-\bar{\beta}_{n} t\right)}{\left(t-\beta_{1}\right) \cdots\left(t-\beta_{n}\right)}=\frac{\left(\frac{1}{t}-\bar{\beta}_{1}\right) \cdots\left(\frac{1}{t}-\bar{\beta}_{n}\right)}{\left(1-\frac{\beta_{1}}{t}\right) \cdots\left(1-\frac{\beta_{n}}{t}\right)}
$$

has the form of the left-hand member of (5.2) and hence approaches zero uniformly with respect to $t$. It follows that the right-hand member of (11.1) approaches zero with $1 / n$, uniformly for $|z| \leqq 1$, and the proof is complete.

THEOREM IX. If the points $\beta_{n}$ have no limit point of modulus greater ihan $1 / A<1$, and if $f(z)$ is analytic for $|z|<T>1$, then the formal expansion (4.1) converges to $f(z)$ for $|z|<\left(A^{2} T+T+2 A\right) /\left(2 A T+A^{2}+1\right)$, uniformly for $|z| \leqq R<\left(A^{2} T+T+2 A\right) /\left(2 A T+A^{2}+1\right)$, except that convergence does not occur at points $1 / \bar{\beta}_{n}$ nor uniform convergence in neighborhoods of such points.

We prove Theorem IX first under the hypothesis $\left|\beta_{n}\right|<1 / A$. We choose $A^{\prime}, 1<A^{\prime}<A$, and $T^{\prime}, 1<T^{\prime}<T$. Return again to (11.1), where $C^{\prime}$ is now the circle $|t|=T^{\prime}$. Equation (11.1) is valid for $z$ interior to $C^{\prime}$. The uniform approach to zero of the right-hand member of (11.1) is assured provided we have $|z|=Z$, 


$$
\frac{A^{\prime} Z-1}{A^{\prime}-Z} \cdot \frac{T^{\prime}+A^{\prime}}{1+A^{\prime} T^{\prime}}<1
$$

or $Z<\left(A^{\prime 2} T^{\prime}+T^{\prime}+2 A^{\prime}\right) /\left(2 A^{\prime} T^{\prime}+A^{\prime 2}+1\right)$; this last quantity is less than $T^{\prime}$. If we now allow $A^{\prime}$ to approach $A$ and $T^{\prime}$ to approach $T$, Theorem IX is established under the hypothesis $\left|\beta_{n}\right|<1 / A$.

Let us again choose $A^{\prime}, 1<A^{\prime}<A$, and choose $N$ such that $n>N$ implies $\left|\beta_{n}\right|<1 / A^{\prime}$. In (9.12) we choose a fixed $n$ greater than $N$, so, as has just been proved, the series (9.13) converges to the function (9.12) uniformly for $|z| \leqq R<\left(A^{\prime 2} T+T+2 A^{\prime}\right) /\left(2 A^{\prime} T+A^{\prime 2}+1\right)$. The expansion (9.13) of the function (9.12) can be written in the form (4.1), which is then uniformly valid (except in neighborhoods of points $\left.1 / \bar{\beta}_{n}\right)$ for $|z| \leqq R<\left(A^{\prime 2} T+T+2 A^{\prime}\right) /\left(2 A^{\prime} T\right.$ $\left.+A^{\prime 2}+1\right)$. If we now allow $A^{\prime}$ to approach $A$, the proof of Theorem IX becomes complete.

The quantity $\left(A^{2} T+T+2 A\right) /\left(2 A T+A^{2}+1\right)$ is greater than unity, and under the hypothesis of Theorem IX can be replaced by no larger quantity.

THEOREM X. If the function $f(z)$ is analytic for $|z|<T<1$ and if the points $\beta_{n}$ have no limit point of modulus greater than $1 / A<T$, then the formal expansion (4.1) of $f(z)$ found by interpolation converges to $f(z)$ for $|z|<\left(A^{2} T-2 A+T\right)$ $/\left(A^{2}-2 A T+1\right)$, uniformly for $|z| \leqq R<\left(A^{2} T-2 A+T\right) /\left(A^{2}-2 A T+1\right)$.

The proof of Theorem $\mathrm{X}$ is quite similar to the proof of Theorem IX; inequality (11.2) is now replaced by

$$
\frac{1+A^{\prime} Z}{A^{\prime}+Z} \cdot \frac{A^{\prime}-T^{\prime}}{A^{\prime} T^{\prime}-1}<1
$$

The details of the proof are left to the reader.* Strangely enough, points $\beta_{n}$ of modulus (less than unity but) greater than $T$ may enter into Theorem X. The function $f(z)$ need not be defined at such points, and the values used for interpolation to $f(z)$ at such points are entirely arbitrary. Series (4.1) nevertheless converges to $f(z)$ as stated, but equation (4.1) need not be valid in all the points $\beta_{n}$, so the coefficients are not uniquely determined.

* It may be noticed that the following theorem can be proved similarly.

If $f(z)$ is analytic for $|z|<T<1$, and if the points $\beta_{i n}, i=1,2, \cdots, n ; n=1,2, \cdots$, have no limit point of modulus less than $1 / A<T$, then the (unique) function

$$
r_{n}(z)=\frac{A_{0 n} z^{n-1}+A_{1 n} z^{n-2}+\cdots+A_{n n}}{\left(1-\bar{\beta}_{1 n} z\right)\left(1-\bar{\beta}_{2 n} z\right) \cdots\left(1-\bar{\beta}_{n n} z\right)}
$$

which coincides with $f(z)$ in the points $\beta_{\text {in }}$, converges to $f(z)$ uniformly for $|z| \leqq R<\left(A^{2} T-2 A+T\right)$ $/\left(A^{2}-2 A T+1\right)$. 
12. Further results; $\prod\left|\beta_{n}\right|$ convergent. We prove the following theorem:

TheOREM XI. Let $\Gamma$ be an arbitrary rectifiable Jordan curve, or set of a finite number of non-intersecting rectifiable Jordan curves bounding a region $R$. Let the product $\Pi\left|\beta_{n}\right|$ be convergent, $\left|\beta_{n}\right|<1$, let the points $\beta_{n}$ lie interior to $R$, and let $f(z)$ be analytic interior to $R$, represented by Cauchy's integral

$$
f(z)=\frac{1}{2 \pi i} \int_{\Gamma} \frac{f_{1}(t) d t}{t-z}, z \text { in } R,
$$

where $f_{1}(t)$ is integrable on $\Gamma$. Suppose finally that no arc of $\Gamma$ either contains a point $1 / \bar{\beta}_{n}$, or, if not a part of $C$, meets $C$ at a limit point of the $\beta_{n}$. Then the formal development (4.1) of $f(z)$ found by interpolation converges for $z$ interior to $R$ except at points and limit points of the $1 / \bar{\beta}_{n}$, uniformly on any closed point set interior to $R$ containing no point $1 / \bar{\beta}_{n}$ or limit point of points $1 / \bar{\beta}_{n}$.

The product $B(z)$ of $\$ 2$ converges at every point $z$ not a point $1 / \bar{\beta}_{n}$ or limit point of points $1 / \bar{\beta}_{n}$, and uniformly on any closed point set containing no point or limit point of the $1 / \bar{\beta}_{n}$. It does not follow that $B_{n}(z)$ approaches $B(z)$ uniformly on $\Gamma$, for $C$ or an arc of $C$ may belong to $\Gamma$. It is not even certain that the values of $B(z)$ defined for $|z| \neq 1,1 / \bar{\beta}_{n}$ by the convergent product, lead to the same boundary values on $C$ for interior and exterior approach.

The function $B(z)$ defined almost everywhere on $C$ as the boundary values of the infinite product $B(z)$ is the same (almost everywhere on $C$ ) as the boundary values $B_{0}(z)$ of the infinite product $B(z)$ defined almost everywhere on $C$ by exterior radial approach.

We have already proved that on $C$ the sequence $B_{n}(z)$ approaches $B(z)$ in the mean. This same reasoning, applied to the function $1 / B(1 / z)$, shows that the sequence $1 / B_{n}(1 / z)$ approaches $1 / B_{0}(1 / z)$ in the mean on $C$. Then by the reasoning already used in $\S 7$, the sequence $B_{n}(z)$ approaches $B_{0}(z)$ in the mean on $C$, so $B(z)$ and $B_{0}(z)$ must be equal almost everywhere on $C$.

As before, we commence with the development of $1 /(t-z)$ and integrate term by term over $\Gamma$ :

$$
\begin{aligned}
& f(z)-r_{n}(z) \\
& =\frac{1}{2 \pi i} \int_{\Gamma} f_{1}(t) \frac{\left(z-\beta_{1}\right) \cdots\left(z-\beta_{n}\right)\left(1-\bar{\beta}_{1} t\right) \cdots\left(1-\bar{\beta}_{n} t\right)}{\left(1-\bar{\beta}_{1} z\right) \cdots\left(1-\bar{\beta}_{n} z\right)(t-z)\left(t-\beta_{1}\right) \cdots\left(t-\beta_{n}\right)} d t, z \text { in } R .
\end{aligned}
$$

It will be noticed that (12.2) is valid even if $R$ contains points $1 / \bar{\beta}_{n}$, and even if $R$ is an infinite region bounded by the finite curve or curves $\Gamma$. On any arc

* This fact is pointed out by Seidel, these Transactions, vol. 34 (1932), pp. 1-21.

$\dagger$ Equation (12.1) cannot be valid for $z=\infty$ unless $f(\infty)=0$. Series (4.1) always represents at infinity a function which vanishes at infinity, unless some $\beta_{n}$ vanishes. 
of $C$, equation (12.2) may be integrated term by term, as we have already shown; our restriction on $\Gamma$ is not intended to exclude the possibility that an arc of $C$ should be an arc of $\Gamma$. On any arc of $\Gamma$ not an arc of $C$, equation (12.2) may also be integrated term by term, for on such an arc the product $B_{n}(z)$ approaches $B(z)$ uniformly, and $1 / B_{n}(t)$ approaches $1 / B(t)$ uniformly. Thus the sequence $r_{n}(z)$ converges to the function

$$
F(z)=f(z)-\frac{B(z)}{2 \pi i} \int_{\Gamma} \frac{f_{1}(t) d t}{(t-z) B(t)}, z \text { in } R .
$$

Convergence occurs at every point of $R$ except points $1 / \bar{\beta}_{n}$ and limit points of such points; convergence is uniform on any closed set interior to $R$ containing no point $1 / \bar{\beta}_{n}$ or limit point of the $1 / \bar{\beta}_{n}$.

There is no assurance that the function $F(z)$ defined by (12.3) is a monogenic* analytic function. If every point of $C$ is a limit point of the $\beta_{n}$, the analytic functions $F(z)$ and $B(z)$ defined interior to $C$ cannot be continued analytically across $C$, nor can the analytic functions $F(z)$ and $B(z)$ defined exterior to $C$ be continued analytically across $C$.

It follows from Theorem XI that whenever $\Pi\left|\beta_{n}\right|$ is convergent and $f(z)$ is analytic in a region containing in its interior all the points $\beta_{n}$ and their limit points, then the formal expansion (4.1) of $f(z)$ converges at every point of analyticity of $f(z)$ except at points and limit points of the $1 / \bar{\beta}_{n}$. Under such restrictions on $f(z)$, it is not necessarily true that $f(z)$ can be represented by (12.1) with a fixed curve or set of curves $\Gamma$ serving simultaneously for all values of $z$. for which $f(z)$ is analytic. Nevertheless for every point $z$ of analyticity of $f(z)$ a suitable curve or set of curves $\Gamma$ (depending on $z$ ) can be found such that (12.1) is valid and $\Gamma$ satisfies the requirements of Theorem XI. Consequently (12.3) is also valid and $F(z)$ is analytic also at $z$ unless $z$ is a point or limit point of the $1 / \bar{\beta}_{n}$.

13. Additional remarks. In the present paper our primary object has been to solve the problem of interpolation in the points $\beta_{n}$ for functions of class $E_{2}$. Nevertheless, a number of other results have been obtained incidentally - for instance, regarding functions of class $E_{p}$-and various other problems are closely related to the present discussion. The writer hopes on another occaion to return to the more detailed treatment of some of these problems. For the present we content ourselves with a few general remarks outlining several such problems.

In particular, it is to be noticed that we have studied in the present paper

* That is, in the sense of Weierstrass. It is quite conceivable that the function $B(z)$ is monogenic in the sense of Borel, and that $F(z)$ is also if $R$ contains $C$ in its interior. 
the analogue of the Taylor expansion; there exists a corresponding analogue of the Laurent expansion which deserves consideration.

Meromorphic functions. Given points $\beta_{1}, \beta_{2}, \cdots$ interior to $C$; let there be prescribed at each point $\beta_{n}$ terms of the form

$$
\begin{aligned}
\frac{\gamma_{-k_{n}}^{(n)}}{\left(z-\beta_{n}\right)^{k_{n}}}+ & \frac{\gamma_{-k_{n}+1}^{(n)}}{\left(z-\beta_{n}\right)^{k_{n}-1}}+\cdots+\gamma_{0}^{(n)}+\gamma_{1}^{(n)}\left(z-\beta_{n}\right)+\cdots \\
& +\gamma_{l_{n}}^{(n)}\left(z-\beta_{n}\right)^{l_{n}}, \quad \gamma_{-k_{n}}^{(n)} \neq 0
\end{aligned}
$$

does there exist a function $f(z)$ possessing boundary values almost everywhere on $C$ which are integrable and with an integrable square on $C$, analytic interior to $C$ except at the points $\beta_{n}$, whose Laurent development about the point $\beta_{n}$ has (13.1) as its first significant $k_{n}+l_{n}+1$ terms? This problem can be completely solved by the results we have already established, provided the product $\prod\left|\beta_{n}\right|^{k_{n}+l_{n}+1}$ converges; the solution is obtained by studying the existence of the function $F(z)$ defined formally by the equation

$$
F(z)=f(z) B(z),
$$

where $B(z)$ is the Blaschke product in which the $\beta_{n}$ appear with the respective multiplicities $k_{n}+l_{n}+1$.

Functions of minimum norm. The solution of the following problem is contained in Theorem VII. Given the points $\beta_{1}, \beta_{2}, \cdots, \beta_{m}$ interior to $C$ and the functional values $\gamma_{1}, \gamma_{2}, \cdots, \gamma_{m}$; to prove the existence of and determine the unique analytic function $f(z)$ of class $E_{2}$ of minimum norm on $C$ which takes on the values $\gamma_{n}$ in the respective points $\beta_{n}$. It can also be shown that if $p_{k}(z)$ is the polynomial of degree $k(=m-1, m, m+1, \cdots)$ of minimum norm on $C$ which takes on the values $\gamma_{n}$ in the points $\beta_{n}$, then we have

$$
\lim _{k \rightarrow \infty} p_{k}(z)=f(z)
$$

uniformly for $z$ on and within $C$; indeed this equation is valid for $z$ interior to the circle of convergence of the Maclaurin development of $f(z)$, uniformly for $z$ on any closed point set interior to this circle of convergence. The writer hopes to consider this problem in more detail on another occasion. The analogous problem of the existence and determination of the function $f(z)$ and of the limit of the sequence of polynomials $p_{k}(z)$ has recently been studied, ${ }^{*}$ where the requirement for $f(z)$ and $p_{k}(z)$ is not that of minimum norm on $C$, but of minimum maximum modulus on $C$. Theorems III and VII solve the present problem (minimum norm on $C$ ) of the existence and determination

* Walsh, these Transactions, vol. 32 (1930), pp. 335-390. 
of $f(z)$ even in the case that the $\beta_{n}$ are infinite in number. When the $\beta_{n}$ are infinite in number, a polynomial $p_{k}(z)$ of degree $k$ cannot necessarily be subjected to the requirement of taking on the values $\gamma_{n}$ in the points $\beta_{n}$, but if $f(z)$ exists and if $p_{k}(z)$ denotes the unique function of class $C_{2}$ of minimum norm on $C$ which takes on the values $\gamma_{1}, \gamma_{2}, \cdots, \gamma_{k+1}$ in the points $\beta_{1}$, $\beta_{2}, \cdots, \beta_{k+1}$, then it can be shown that the equation

is valid for $|z|<1$.

$$
\lim _{k \rightarrow \infty} p_{k}(z) .=f(z)
$$

Normal families. In the study of the various phases of the problem just mentioned, two theorems relating to normal families of functions are particularly useful.

If the functions $f^{(n)}(z)$ are all of class $E_{2}$, if we have

$$
f^{(n)}(z)=\frac{1}{2 \pi i} \int_{c} \frac{f_{1}^{(n)}(t) d t}{t-z},|z|<1,
$$

where the functions $f_{1}^{(n)}(z)$ are integrable together with their squares on $C$, and if we have

$$
\int_{C}\left|f_{1}^{(n)}(t)\right|^{2}|d t| \leqq M,
$$

where $M$ is independent of $n$, then the functions $f^{(n)}(z)$ form a normal family interior to C. Any limit function of this family is of class $E_{2}$ and can be expressed by

$$
f(z)=\frac{1}{2 \pi i} \int_{C} \frac{f_{1}(t) d t}{t-z}
$$

where $f_{1}(t)$. (boundary values of $f(z)$ defined almost everywhere on $C$ by radial approach) is integrable together with its square on $C$, and we have

$$
\int_{C}\left|f_{1}(t)\right|^{2}|d t| \leqq M .
$$

The next theorem is an application of Theorem IV.

If a family of functions $f_{n}(z)$ analytic and forming a normal family interior to $C$ has no limit function interior to $C$ not of class $E$, then every subsequence of the family which converges on a point set $\beta_{n}$, where $\prod\left|\beta_{n}\right|$ diverges, converges interior to $C$, uniformly for $|z| \leqq r<1$.

It is worth noticing that in this theorem (a generalization of the theorems of Vitali and of Blaschke) the points $\beta_{n}$ need not be all distinct and indeed may 
all coincide, provided we make the convention usual in the present paper, that the condition

$$
\lim _{n \rightarrow \infty} f_{n}(\beta) \text { exists, }
$$

where $k$ points $\beta_{n}$ coincide at $\beta$, is intended to imply the conditions on the derivatives

$$
\lim _{n \rightarrow \infty} f_{n}^{(m)}(\beta) \text { exists } \quad(m=0,1,2, \cdots, k-1) .
$$

If an infinity of points $\beta_{n}$ coincide at $\beta$, this condition involves all the derivatives:

$$
\lim _{n \rightarrow \infty} f_{n}^{(m)}(\beta) \text { exists } \quad(m=0,1,2, \cdots) \text {; }
$$

condition (13.2) is precisely the condition for convergence of the sequence $f_{n}(z)$ in the point $\beta$, counted of infinite multiplicity, and is itself sufficient to imply the convergence of the sequence $f_{n}(z)$ interior to $C$, uniformly for $|z| \leqq r<1$.

Other series of interpolation. A generalization of series (1.1) and (4.1) is the series

$$
a_{0} \phi_{0}(z)+a_{1}\left(z-\beta_{1}\right) \phi_{1}(z)+a_{2}\left(z-\beta_{1}\right)\left(z-\beta_{2}\right) \phi_{2}(z)+\cdots,
$$

and this series, where the $\phi_{n}(z)$ are suitably thosen, has much in common with the series (1.1) and (4.1).

HARVARD UNIVERSITY,

Cambridge, Mass. 